\title{
Improvement in the Extraction of Hass Avocado Virgin Oil by Ultrasound Application
}

\author{
Nadia Segura $^{1}$, Miguel Alejandro Amarillo ${ }^{1}$, Natalia Ines Martinez ${ }^{1} \&$ María Antonia Grompone $^{1}$ \\ ${ }^{1}$ Área Grasas y Aceites, Depto CYTAL, Facultad de Química, Univerisdad de la República, Montevideo, \\ Uruguay \\ Correspondence: Miguel Alejandro Amarillo. Área Grasas y Aceites, Depto CYTAL, Facultad de Química, \\ Univerisdad de la República, Montevideo, Uruguay. E-mail: amarillom@fq.edu.uy
}

Received: December 11, 2017

Accepted: December 29, 2017 Online Published: February 23, 2018

doi:10.5539/jfr.v7n2p106

URL: https://doi.org/10.5539/jfr.v7n2p106

\begin{abstract}
The virgin oil extraction from avocado Hass was carried using an Abencor pilot scale plant.

High-power ultrasound (1.73 MHz) was applied after mixing. High-frequency ultrasound device consists of two transductors which each deliver $30 \mathrm{~W} / \mathrm{L}$.

Different proportions of water were added before ultrasound treatment (no water added and paste:water relation $(1: 3,1: 2,1: 1,2: 1,3: 1)$ and also different ultrasound application times $(0,1,2,3,4,5,10,15,20$ and 25 minutes). The study was carried out by setting one of the two variables considered and changing the values of the other. In the water addition study, ultrasound time was set at $15 \mathrm{~min}$.

It was found that oil recovery increased with the percentage of water added. It was decided to employ a ratio of 1:1 to study the influence of ultrasound application time. Under these conditions, it was found that with 1 minute of ultrasound application, recovery increased by $40 \%$ over the process without ultrasound.

It was observed that the composition in fatty acids and the content of natural antioxidants (tocopherols and polyphenols) are not affected by the ultrasound application.

It is concluded that the application of high-frequency ultrasound with addition of water post malaxing improves recovery of virgin avocado oil without negative effects on the general quality of the oil.
\end{abstract}

Keywords: extraction, avocado oil, extra virgin, ultrasound.

\section{Introduction}

\subsection{Introduce the Problem}

Several authors studied the application of ultrasound in food (Jiménez et al., 2006) and its effects on oils (Chemat et al., 2004; Chemat et al., 2004; Cañizares-Macías et al., 2004; Sharma \& Gupta, 2006; Benedito et al., 2007; Zhang et al., 2008; Jerman et al., 2010; Izbaima et al., 2010; Riera et al., 2010; Li et al., 2004; Liu et al., 2011; Jerman \& Mozetic, 2012; Da Porto et al., 2013; Goula, 2013; Tian et al., 2013; Rodríguez et al., 2013; Reboredo-Rodríguez, 2014; Samarama et al., 2014).

Ultrasound has been applied in the production of palm oil. Mechanical vibration, acoustic flow and cavitation result in large localized forces that produce physical changes such as surface tension reduction and clustering particle density. Water sonication includes forming hydroxyl radicals. The mechanical effects dominate at low frequencies $(20-80 \mathrm{kHz})$ and at frequencies higher than $1500 \mathrm{kHz}$; chemical effects take place from 100 to 1000 kHz. Juliano et al. (2013), applied frequency between 20-2000 kHz. The Malaysian company Tai Tak Snd Bhd incorporated this process industrially achieving an annual increase of $1 \%$ in the production of crude palm oil with an additional gain of approximately US $\$ 1,000,000$.

Avocado (Persea americana Mill.) is a mexican origin specie that belongs to the family of Lauraceae. This family includes about 150 species, most of which grow in tropical America. Avocado pulp contains more than 20\% oil. For example, a fruit Hass contains about 200 grams of pulp and, therefore, about 40 grams of oil.

The oil extracted from the pulp of the avocado has many applications. Yanty et al. (2012) studied the composition of avocado oils from different varieties. The food industry uses it to prepare canned foods and salad 
dressings. The cosmetics industry uses it in the formulation of lotions, creams and soaps for skin care and hair care. The pharmaceutical industry uses it as a base for ointments, salves and balms. Currently they are studied other ways to use it in medicines and nutraceuticals. It's international price is very expensive so it is justified to study changes in the extraction procedure in order to increase their performance.

In this paper the application of high frequency ultrasound $(1,73 \mathrm{MHz})$ was optimized in order to increase the extraction yield of virgin avocado oil. The qualities of the oils obtained in the Abencor pilot scale plant with and without the high-frequency ultrasound application were compared

\section{Method}

\subsection{Raw Material}

For this study, Chilean origin avocado from "Hass" variety, acquired in the local market in a state of maturity appropriate for their intake were used. This optimum maturity considered is observed when the skin is dark and the fruit is firm but not hard to the touch.

As maturity varies with post-harvest time, avocados cannot be stored for many days. It was intended that all lots had the same maturity degree. Despite these precautions, the lots presented differences in composition (amount of oil and moisture from the pulp). As the oil pulp content from different batches is not constant, it was necessary to define a coefficient of Performance (CP), which considers the relation between the mass of oil obtained for each test on the mass of oil obtained from control, bearing in mind the same average sample of ground pulp (equation 1):

$$
\text { C.P }=\frac{\text { mass of oil obtained }}{\text { mass of oil control }}
$$

\subsection{Oil Extraction of Avocado Pulp in an Abencor Pilot Scale Plant}

The extraction of virgin avocado oil was performed in an Abencor pilot scale plant for extracting virgin olive oil. First, the avocados were manually peeled and their seed separated in order to obtain only the pulp. The oil extraction begins with grinding the pulp to obtain a paste by employing a hammer mill, followed by a malaxation step at a defined temperature and finally a vertical centrifuge for separating the liquid phase (oil and water) from the solid phase (rest of the pulp). Instead of the Abencor hammer mill, an electric food processor for grinding the avocado pulp was employed. The next step was the paste malaxation in a Thermo-mixer TB-100 at $40{ }^{\circ} \mathrm{C}$ for 6 hours, with addition of water in a ratio 5:1 paste: water at 4,5 hours of mixing, to favor the separation of oil from paste. Finally the paste was centrifuged in a Centrifugal machine CF-100 at $3500 \mathrm{rpm}$ for 1 minute.

\subsection{Application of Ultrasound in Oil Extraction of Avocado Pulp in an Abencor Pilot Scale Plant}

Ultrasound was applied to the pulp at a frecuency of $1,73 \mathrm{MHz}$ between the malaxing and centrifugation steps.

The ultrasound device consists of two transducers which deliver a power of $30 \mathrm{~W} / \mathrm{L}$ each. The tests were conducted using a single transducer. The container vessel is a square prism $(7.5 \times 7.5 \times 18 \mathrm{~cm})$ with a $1000 \mathrm{~mL}$ capacity. A volume of $500 \mathrm{~mL}$ paste was added, so that only one transducer was employed. After the application of ultrasound, pulp was centrifuged in a centrifuge SORVALL SS-4 at $3500 \mathrm{rpm}$ for 15 minutes. The Abencor system centrifuge could not be used because it was not effective due to the high fluidity of the paste, as a consequence of the amount of water added.

2.3.1 Influence of Adding Different Proportions of Water on Yield of Extracted Oil (For the Same Ultrasound Application Time)

To study the possible increase in oil extraction, different proportions of water were added to the paste prior to application of ultrasound. The obtained results were compared to those obtained from the extraction process in the Abencor plant described in section 2.2 (without addition of water and without application of ultrasound, called "control"). The paste:water relations used were 1:3, 1:2, 1:1, 2:1, 3:1 and non water addition (maintaining constant application time ultrasound in 15 minutes).

2.3.2 Influence of Ultrasound Application Time on Yield of Extracted Oil (For the Same Amount of Water Added)

The relation paste:water (1:1) was selected as the most appropriate for the different ultrasound application times. The application times evaluated were: 5, 10, 15, 20 and 25 minutes. The results were compared with the control sample obtained as described in 2.2. In a second step, shorter times of ultrasound application were studied (1, 2, 3,4 and 5 minutes) as a possibility for improving the industrial application. 


\subsection{Analytical Techniques for the Characterization of Oils}

\subsubsection{Oil Content of Avocado Pulp by Soxhlet Method}

The oil content was determined on the dry pulp by the Soxhlet method using petroleum ether $\left(62-68{ }^{\circ} \mathrm{C}\right)$ for analysis during 8 hours. Prior to this solvent extraction the sample was dried in an oven drying with forced convection at $55.0 \pm 0.5^{\circ} \mathrm{C}$ for 12 hours.

\subsubsection{Biophenols Content}

According to International referee method COI/T.20/Doc. ${ }^{\circ} 29$, "Determination of biophenols from olive oils by HPLC".

\subsubsection{Tocopherol Content By HPLC}

According to Andrikopoulos et al. (1991) method.

\subsubsection{Acidity Value}

According to standard method IUPAC 2.201.

\subsubsection{Peroxide Value}

According to standard method IUPAC 2.501.

\subsubsection{Absorbency in Ultra-violet (K232 and K270)}

According to International referee method COI/T.20/Doc. $\mathrm{n}^{\circ}$ 19/Rev. 3, "Spectrophotometric investigation in the ultraviolet".

\subsubsection{Fatty Acid Composition}

The preparation of the methyl esters was according to official method AOCS Ch 1-91 and its determination by gas chromatography according to official method AOCS Ce 1e-91.

\subsubsection{Chlorophyll Pigments Content in Crude Vegetable Oils}

According to official method AOCS Cc 13i-96. The content of chlorophyll pigments in vegetable oils is expressed as $\mathrm{mg}$ of pheophytin a in $1 \mathrm{Kg}$ of oil.

\subsubsection{Statistical Analysis}

The data were treated statistically by analysis of variance (ANNOVA) and using the Tukey test of INFOSTAT with a significancy level of 0.05 .

\section{Results and Discussion}

3.1 Influence of Adding Different Proportions of Water on Yield of Extracted Oil (For the Same Ultrasound Application Time)

The results of CP based on paste:water relation are shown in Figure 1. The CP was not increased by applying ultrasound without addition of water nor for the relation 3:1. This could be due to the fact that this type of paste does not allow the propagation of waves within it making ineffective the application of ultrasound. By increasing the amount of water the CP increased dramatically for relations paste: water 2:1, 1:1, 1:2 and 1:3, among which there was no significant difference.

While higher CP was obtained with relation paste:water 1:3, at industrial scale it is better to work with a paste:water ratio 1:1 because of the smaller amount of water required. This involves a reduced capacity of equipment and less effluent volumes to be treated. The CP value of relation paste: water 2:1 showed no significative difference with the corresponding value ratio of 1:1. 


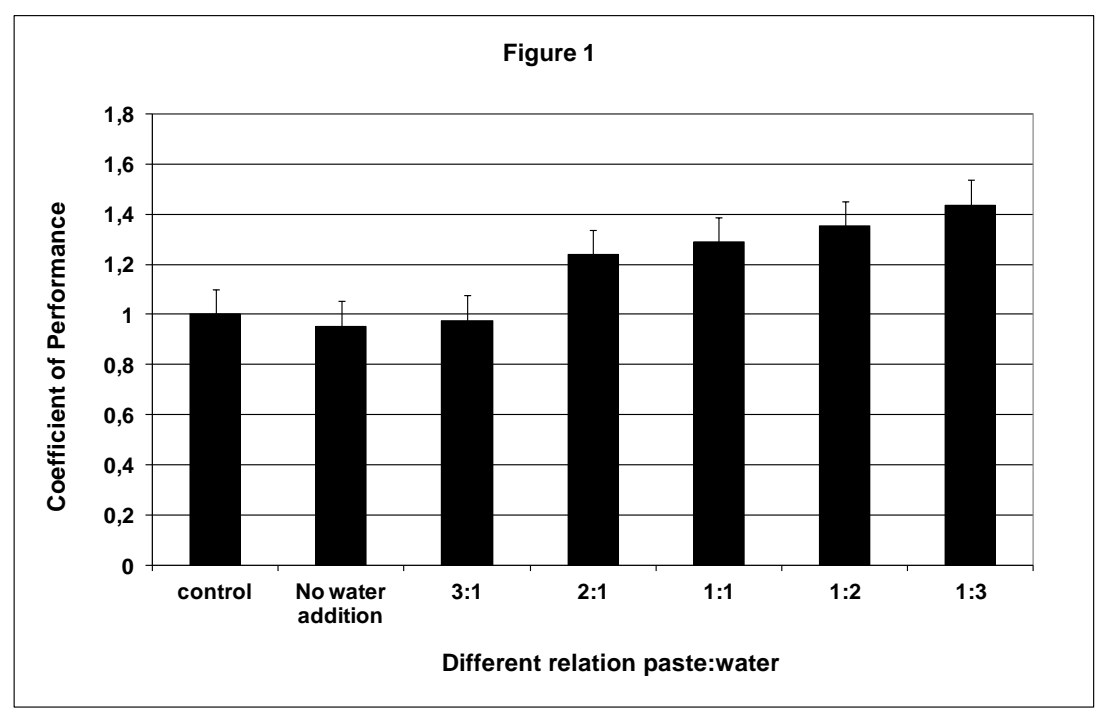

Figure 1. Coefficient of performance as function of different relation paste:water, expressed with it's margin of error

\subsection{Influence of Ultrasound Application Time on Yield of Extracted Oil (For a Paste: Water 1:1 Relation)}

The CP results according to ultrasound application times 5, 10, 15, 20 and 25 minutes are shown in Figure 2. With 5 minutes of application the CP increased $33 \%$ over the process without application of ultrasound. There was not a significant increase in the amount of extracted oil by prolonging the ultrasound application time (taking into account the margin of error shown in Figure 2).

According to these results smaller application times (1, 2, 3, 4 and $5 \mathrm{~min}$ ) were evaluated. The results are shown in Figure 3. The $\mathrm{CP}$ increased for all application times compared to the control sample. There was no difference among these application times (to take into account the margins of error shown in Figure 3).

It was observed that only one minute of ultrasound application was enough for increasing oil extractability in $40 \%$ $(\mathrm{CP}=1.4)$ under the studied conditions (Figure 1). Martinez-Padilla et al. (2018), studied ultrasound application $(2 \mathrm{MHz})$ in avocado puree prior to malaxing step without water addition, they obtained an increase of $15-24 \%$ in oil recovery. Further studies must be carried on in order to get a better understanding of ultrasound and it's application in food industry, but both researches show how usefull high frequency ultrasound proves to be in oil extraction proccesses such as avocado oil.

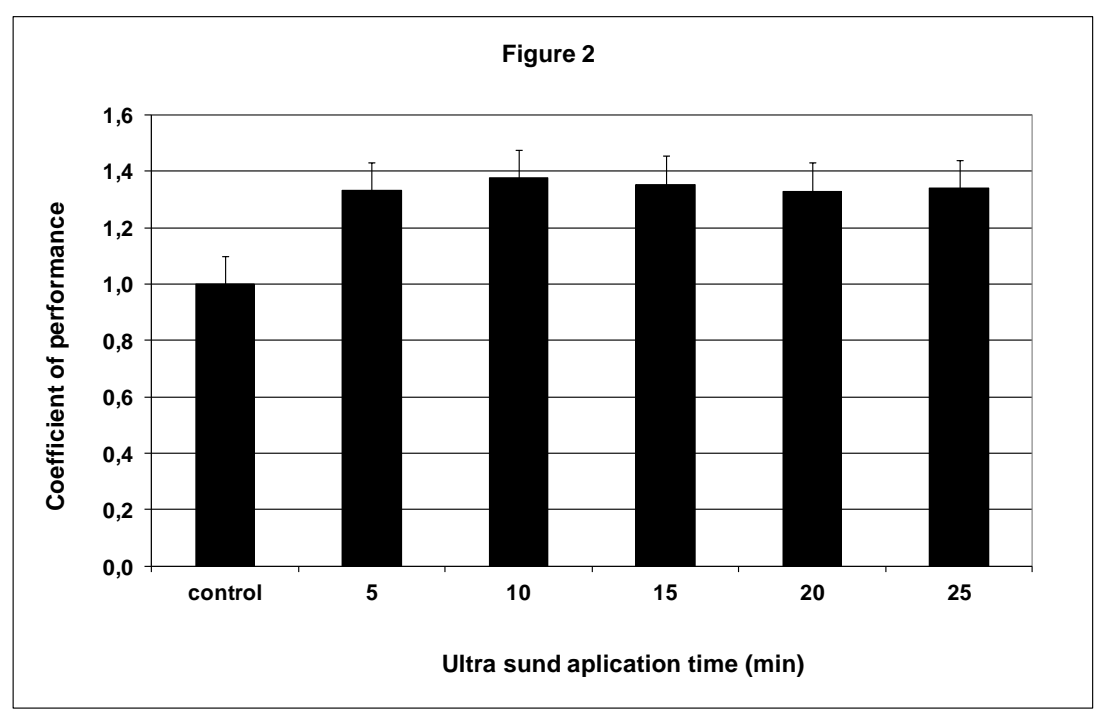

Figure 2. Coefficient of performance as function of different ultrasound application time with relation paste:water 1:1, expressed with it's margin of error 


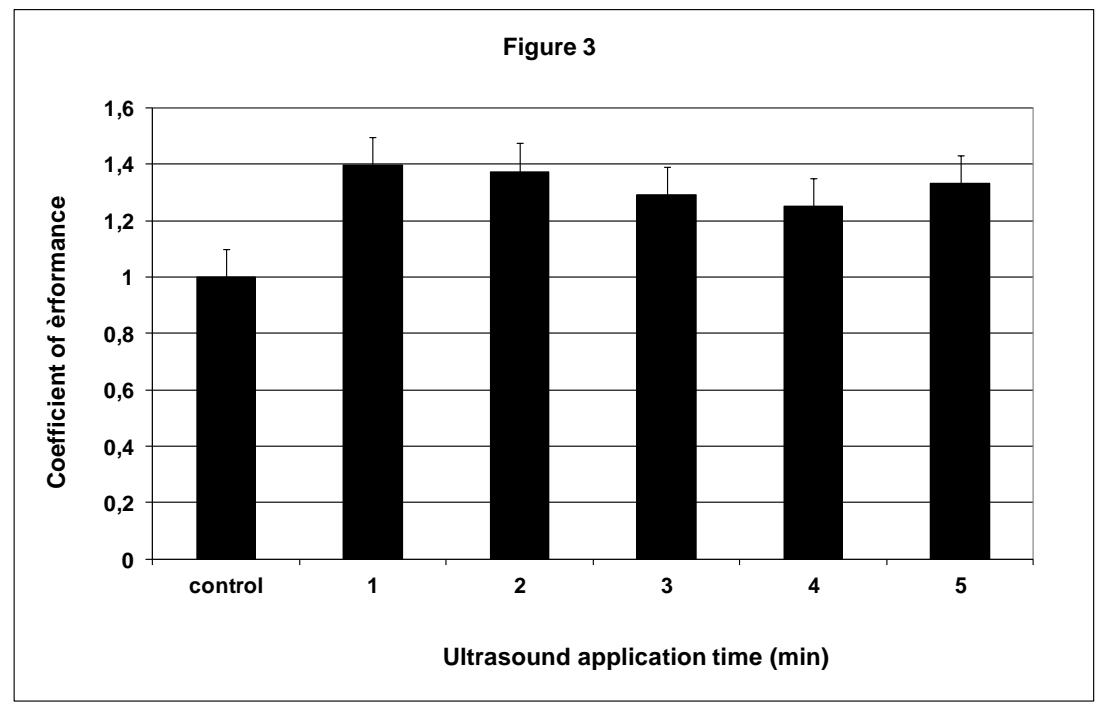

Figure 3. Coefficient of performance as function of different ultrasound application time with relation paste:water $1: 1$, expressed with it's margin of error

\subsection{Influence of the Ultrasound Application on the Purity and Quality of Extracted Avocado Oil}

The composition in fatty acids was studied as criterion of nutritional quality and oxidative stability of the oils. The fatty acid profile of the oils extracted by, Soxhlet method and Abencor pilot plant, with and without ultrasound application was evaluated from a single batch of avocado, the results are observed in Table 1.

Table 1. Composition in fatty acids of the oil of avocado extracted by Soxhlet method and in pilot plant with and without the application of ultrasound (US)

\begin{tabular}{cccc}
\hline & \multicolumn{2}{c}{ Abencor } \\
\hline & Soxhlet & with US & without US \\
\hline $16: 0$ & 20.7 & 21.2 & 21.2 \\
$16: 1$ & 11.5 & 11.8 & 11.9 \\
$18: 0$ & 0.5 & 0.4 & 0.4 \\
$18: 1$ & 51.3 & 52.2 & 51.9 \\
$18: 2$ & 12.8 & 13.0 & 13.1 \\
$18: 3$ & 0.6 & 0.6 & 0.6 \\
\hline
\end{tabular}

It is observed that the extraction method does not affect the fatty acid profile of the oil. It was found that the main fatty acid was oleic acid (52\%) followed by palmitic (21\%). Therefore it can be said that this oil has good oxidative stability considering the fatty acids composition.

Table 2 shows the contents of natural antioxidants (tocopherols and phenols), acidity, peroxide value, absorbancy in ultra-violet and pheophytin a content of extracted oils.

Table 2. Content of tocopherols and phenols, acidity value, peroxide value, absorbency in ultra-violet and pheophytin a content of avocado oil extracted in Abencor plant with and without ultrasound application.

\begin{tabular}{lll}
\hline & with US & without US \\
\hline$\alpha$-Tocopherols (ppm) & $412 \pm 2^{\mathrm{a}}$ & $357 \pm 44^{\mathrm{a}}$ \\
$\beta+\gamma$-Tocopherols (ppm) & $59 \pm 1^{\mathrm{a}}$ & $58 \pm 0^{\mathrm{a}}$ \\
$\delta$-Tocopherols (ppm) & $8 \pm 0^{\mathrm{a}}$ & $9 \pm 0^{\mathrm{a}}$ \\
Phenols (ppm) & $54 \pm 5^{\mathrm{a}}$ & $59 \pm 3^{\mathrm{a}}$ \\
Acidity (\%) & $0.33 \pm 0.02^{\mathrm{a}}$ & $0.52 \pm 0.01^{\mathrm{b}}$ \\
PV (meq O $/ \mathrm{Kg})$ & $8.06 \pm 0.27^{\mathrm{a}}$ & $9.64 \pm 0.31^{\mathrm{b}}$ \\
K232 & $2.68 \pm 0.02^{\mathrm{a}}$ & $3.13 \pm 0.16^{\mathrm{a}}$ \\
K268 & $0.20 \pm 0.01^{\mathrm{a}}$ & $0.19 \pm 0.02^{\mathrm{a}}$ \\
Pheophytin a (mg/kg oil) & $35.5 \pm 0.1^{\mathrm{a}}$ & $33,2 \pm 0,2^{\mathrm{b}}$ \\
\hline
\end{tabular}

Note. Different letters in the same column indicate significant differences according to Tukey's test $(\mathrm{p}<0.05)$. 
It was observed that the main antioxidants content in virgin avocado oil are tocopherols, in particular $\alpha$-tocopherol. Although the obtained oils contain a certain amount of the phenolic type, they are the minority. Clearly there are no significant differences between the amount of all antioxidants for oils extracted with and without application of ultrasound. Therefore, ultrasound application after malaxing of avocado paste did not affect the total content of antioxidants.

It is observed that the acidity value is lower in the oil extracted with ultrasound application. The acidity values are lower comparing to those values for the extra virgin olive oil quality. This is why it could be classified as extra virgin according to Trade Standard applying to olive oils and olive pomace oils of International Olive Council.

A decrease in the peroxide value was also found, suggesting that the application of ultrasound does not compromise the oil quality. This can also be observed in the K232 coefficient. The decrease in the K232 coefficient could be due to the decomposition of the peroxides during the process of application of ultrasound, although the products formed would not absorb at $268 \mathrm{~nm}$, since no changes in the value of K268 were found.

As for chlorophyll pigments, a slight increase in the content of pheophytin a was observed with the ultrasound application. This could be reflected in the partial loss of the characteristic green color of chlorophyll, with the appearance of a yellowish hue in the oil. This could slightly affect the visual characteristics of the product.

\section{Conclusions}

The application of high frequency ultrasound $(1.73 \mathrm{MHz})$ proved to be an effective tool to increase the extraction yield of virgin avocado oil (Hass variety) when water is added to the paste in a 1:1 relation after the thermo-mixing process at $40{ }^{\circ} \mathrm{C}$. It was found that 1 minute of application of ultrasound was enough to increase the extraction of oil by $40 \%$.

Also, this technology generates partial changes in regards to the quality of avocado oil. It causes a decrease in the acid and peroxide values, while the content of pheophytin a increases due to the decomposition of chlorophyll pigments.

Respect to the antioxidants content, this technology does not affect it, so it could be expected not to modify the oil oxidative stability. It also does not change the fatty acid composition. Consequently, the application of high frequency ultrasound benefits the extraction of avocado oil.

\section{Acknowledgments}

To the Sectoral Commission for Scientific Research (CSIC) in the modality of initiation to research 2015 for the financing of the work presented.

To Ph.D. Pablo Juliano (CSIRO) for supplying the ultrasound equipment and Ph.D. Nicolás Pérez for supporting this research.

And we would especially like to remember Dra. María Antonia Gropone for her invaluable contributions, advice and motivation. RIP

\section{References}

Andrikopoulos, N. K., Brueschweiler, H., Felber, H., \& Taeschler, Ch. (1991). HPLC analysis of phenolic antioxidants, tocopherols and triglycerides. J. Am. Oil Chem. Soc., 68, 359-364. https://doi.org/10.1007/BF02663750

AOCS (1997). Official methods and recommended practices of the American Oil Chemists' Society. Champaign, USA: American Oil Chemists' Society Press.

Benedito, J., García-Pérez, J. V., Dobarganes, M. C., \& Mulet, A. (2007). Rapid evaluation of frying oil degradation using ultrasonic technology. Food Res. Int., 40, 406-414. https://doi.org/10.1016/j.foodres.2006.10.017

Cañizares-Macías, M. P., García-Mesa, J. A., \& Luque de Castro, M. D. (2004). Fast ultrasound-assisted method for the determination of the oxidative stability of virgin olive oil. Analytica Chimica Acta, 502, 161-166. https://doi.org/10.1016/j.aca.2003.10.022

Chemat, F., Grondin, I., Costes, P.; Moutoussamy, L., Shum Cheong Sing, A., \& Smadja, J. (2004). High power ultrasound effects on lipid oxidation of refined sunflower oil. Ultrasonics Sonochemistry, 11, 281-285. https://doi.org/10.1016/j.ultsonch.2003.07.004

Chemat, F., Grondin, I., Shum Cheong Sing, A., \& Smadja, J. (2004). Deterioration of edible oils during food 
processing by ultrasound. Ultrasonics Sonochemistry, 11, 13-15.

https://doi.org/10.1016/S1350-4177(03)00127-5

Da Porto, C., Porretto, E., \& Decorti, D. (2013). Comparison of ultrasound-assisted extraction with conventional extraction methods of oil and polyphenols from grape (Vitis vinifera L.) seeds. Ultrasonics Sonochemistry, 20, 1076-1080. https://doi.org/10.1016/j.ultsonch.2012.12.002

Goula, A. W. (2013). Ultrasound-assisted extraction of pomegranate seed oil. Kinetic modeling. J. Food Eng., 117, 492-498. https://doi.org/10.1016/j.jfoodeng.2012.10.009

IOC (International Olive Council). (2015). Chemistry/Testing Methods/Chemical testing methods Retrieved from http://www.internationaloliveoil.org

IUPAC (1992). International Union of Pure and Applied Chemistry. Standard methods for the analysis of oils, fats and derivatives, 7th Edition. Methods: 2.201 and 2.501. Oxford. Ed.: Pergamon Press.

Izbaima, D., Faiza, B., Moudden, A., Taific, N., \& Aboudaouda, I. (2010). Evaluation of the performance of frying oils using an ultrasonic technique. Grasas y Aceites, 61(2), 151-156. https://doi.org/10.3989/gya.087709

Jerman, T., Trebše, P., \& Mozetic Vodopivec, B. (2010). Ultrasound-assisted solid liquid extraction (USLE) of olive fruit (Olea europaea) phenolic compounds. Food Chem., 123, 175-182. https://doi.org/10.1016/j.foodchem.2010.04.006

Jerman Klen, T., \& Mozetic Vodopivec, B. (2012). Optimization of olive oil phenol extraction conditions using a high-power probe ultrasonication. Food Chem., 134, 2481-2488. https://doi.org/10.1016/j.foodchem.2012.04.096

Jiménez Márquez, A., Beltrán Maza, G., Uceda Ojeda, M., \& Aguilera Herrera, M. P. (2006). Empleo de ultrasonidos de potencia en el proceso de elaboración del aceite de oliva virgen. Resultados a nivel de planta de laboratorio. Grasas y Aceites, 57(3), 253-259.

Juliano, P., Swiergon, P., Mawson, E., Knoerzer, K., \& Augustin, M. A. (2013). Application of ultrasound for oil separation and recovery of palm oil. J. Amer. Oil Chem. Soc., 90, 579-588. https://doi.org/10.1007/s11746-012-2191-y

Li, H., Pordesimo, L., \& Weiss, J. (2004). High intensity ultrasound-assisted extraction of oil from soybeans. Food Res. Int., 37, 731-738. https://doi.org/10.1016/j.foodres.2004.02.016

Liu, S., Jiang, L., \& Li, Y. (2011). Research of aqueous enzymatic extraction of watermelon seed oil of ultrasonic pretreatment assisted. Proc. Eng., 15, 4949 -4955. https://doi.org/10.1016/j.proeng.2011.08.921

Martínez-Padilla, L.P., Franke, L., Xu, X.-Q., \& Juliano, P. (2018). Improved extraction of avocado oil by application of sono-physical processes. Ultrasonics Sonochemistry, 40, 720-726. https://doi.org/10.1016/j.ultsonch.2017.08.008

Reboredo-Rodríguez, P., Rey-Salgueiro, L., Regueiro, J., González-Barreiro, C., Cancho-Grande, B., \& Simal-Gándara, J. (2014). Ultrasound-assisted emulsification-microextraction for the determination of phenolic compounds in olive oils. Food Chem., 150, 128-136. https://doi.org/10.1016/j.foodchem.2013.10.157

Riera, E.; Blanco, A., García, J., Benedito, J., Mulet, A., Gallego-Juárez, J. A., \& Blasco, M. (2010). High-power ultrasonic system for the enhancement of mass transfer in supercritical $\mathrm{CO} 2$ extraction processes. Ultrasonics, 50, 306-309. https://doi.org/10.1016/j.ultras.2009.09.015

Rodriguez, J., Melo, E. C., Mulet, A., \& Bon, J. (2013). Optimization of the antioxidant capacity of thyme (Thymus vulgaris L.) extracts: Management of the convective drying process assisted by power ultrasound. J. Food Eng., 119, 793-799. http://doi.org/10.1016/j.jfoodeng.2013.07.016

Samarama, S., Mirhosseinia,H., Tana, Ch. P., \& Ghazaliba, H. M. (2014). Ultrasound-assisted extraction and solvent extraction of papaya seed oil: Crystallization and thermal behavior, saturation degree, color antioxidative stability. Ind. Crops Prod., 52, 702- 708. http://doi.org/10.1016/j.indcrop.2013.11.047

Sharma, A., \& Gupta, M. N. (2006). Ultrasonic pre-irradiation effect upon aqueous enzymatic oil extraction from almond and apricot seeds. Ultrasonics Sonochemistry, 13, 529-534. https://doi.org/10.1016/j.ultsonch.2005.09.008

Tian, Y., Xu, Z., Zheng, B., \& Lo, Y. M. (2013). Optimization of ultrasonic-assisted extraction of pomegranate 
(Punica granatum L.) seed oil. Ultrasonics Sonochemistry, 20, 202-208. https://doi.org/10.1016/j.ultsonch.2012.07.010

Yanty, N. A. M., Marikkar, J. M. N., \& Miskandar, M. S. (2012). Comparing the termo-physical characteristic of lard and selected plant fats. Grasas y Aceites, 63(3), 328-334. https://doi.org/10.3989/gya.023712

Zhang, Z.-S., Wang, L.-J., Li, D., Jiao, S.-S., Chena, X. D., \& Mao, Z.-H. (2008). Ultrasound-assisted extraction of oil from flaxseed. Sep. Pur. Technol., 62, 192-198. https://doi.org/10.1016/j.seppur.2008.01.014

\section{Copyrights}

Copyright for this article is retained by the author(s), with first publication rights granted to the journal.

This is an open-access article distributed under the terms and conditions of the Creative Commons Attribution license (http://creativecommons.org/licenses/by/4.0/). 